\title{
ARTIGO TÉCNICO \\ METODOLOGIA PARA CÁLCULO RÁPIDO DAS DIMENSÕES \\ DE PNEUS DE TRATORES AGRÍCOLAS
}

\section{LUIS MÁRQUES ${ }^{1}$}

RESUMO: Os primeiros tratores agrícolas utilizavam como elementos de locomoção rodas metálicas com garras. Diante da impossibilidade de circular por estradas, os danos provocados sobre os caminhos e o recalque em solo desagregado tornaram necessária a busca de outros materiais para os rodados dos tratores. Os rodados pneumáticos, ao se deformarem, adaptam-se melhor às irregularidades do terreno, aumentando a capacidade trativa e a comodidade para o operador. A diminuição da lastragem, substituída por cargas dinâmicas induzidas por transferência de peso, melhora ainda mais o desempenho das rodas no solo, convertendo-as em verdadeiros pontos de apoio. Apesar de toda evolução experimentada pelos rodados, os mesmo oferecem diversas opções técnicas que nem sempre são aproveitadas, pois nem todos os rodados disponíveis no mercado são apropriados para qualquer aplicação. $\mathrm{O}$ tamanho, as características técnicas, a pressão de inflação, etc. determinam o comportamento e a durabilidade. Assim, um rodado sobrecarregado tem pouca vida útil; por outro lado, o pensamento contrário onera o produto, uma vez que a falta de peso pode chegar a prejudicar sua função precípua, ressaltando-se que, ao não apoiar toda a banda de rodagem, pode-se aumentar a patinagem. Pelo exposto, torna necessário selecionar adequadamente o rodado para determinada aplicação e procurar que seu uso em cada momento, ocorra em condições que proporcionem melhores benefícios e maio durabilidade.

PALAVRAS-CHAVE: pneus agrícolas; eficiência em tração, patinagem.

\section{METHODOLOGY FOR FAST CALCULATION OF AGRICULTURAL TRACTORS TIRES' DIMENSIONS}

\begin{abstract}
Primary agricultural tractors used metallic wheels with clutches as locomotion elements. Due to the impossibility to run in roads, damages provoked on the pathways and the suppression in disintegrated soil made necessary to seek other materials for the tractors' tread. The pneumatic tread, by being deformed, adjust better to irregularities on the ground, increasing the traction capacity and commodity for the operator. The decreased ballastage, replaced by dynamic loads induced by weight transference, improve even more the performance of the wheels in the soil, converting them in actual support points. In spite of the evolution experienced by the treads, they offer several technical options that aren't always used, because not every tread available in the market are appropriate for every application. The size, technical features, inflation pressure, etc. determine the behavior and durability. Thus, an overloaded tread has a decreased useful life; on the other hand, thinking otherwise charges the product, once the lack of weight can jeopardize its primary function, stressing that, by not supporting the whole tread, gliding can be increased. In consequence of the exposed above, it becomes necessary to choose the tread properly in order to determine the application and find out that its use for each moment occurs in conditions that provide better benefits and higher durability.
\end{abstract}

KEYWORDS: agricultural tires; traction efficiency, gliding.

\footnotetext{
${ }^{1}$ Ing. Agrónomo, Dr., Grupo Investigación: Tractores y Laboreo, Universidad Politécnica de Madrid.

Recibido por el Consejo Editorial en: 14-4-2008

Aprobado por el Consejo Editorial en: 3-10-2008
} 


\section{MODELOS MATEMÁTICOS QUE EXPLICAN EL COMPORTAMIENTO DE LA RUEDA}

Las condiciones del suelo en el que trabajan los tractores y máquinas agrícolas ofrecen una gran variabilidad espacial y temporal. El contenido de agua en el suelo cambia completamente su comportamiento mecánico. Además, los tractores agrícolas también circulan por carreteras y caminos estabilizados, que no se deforman por el paso del neumático.

Cualquier neumático debe de cumplir, de manera general, las siguientes funciones:

- Bajas pérdidas por fricción en la rodadura continúa del vehículo;

- Distribución uniforme de cargas;

- Adaptación a superficies irregulares;

- Transmisión del par motor al suelo con bajo consumo de combustible;

- Aplicación continua y uniforme de las fuerzas de frenada y

- Asegurar la estabilidad lateral del vehículo.

Hay una clara diferencia entre el comportamiento de la rueda cuando se mueve sobre una superficie firme, como la de una carretera, a cuando se mueve en un suelo natural. Analizando el problema en su aspecto más general, cuando se circula por terreno natural, la complejidad de los fenómenos que se producen en la zona de contacto entre la rueda (o la cadena) y el suelo son consecuencia de:

- El hundimiento del terreno bajo la rueda apareciendo en éste una rodada.

- Rotura limitada del terreno como consecuencia de la energía desarrollada. Una rotura excesiva impide el avance.

- Las deformaciones son muy grandes y se producen de forma casi instantánea.

Parte de la energía necesaria para conseguir que la rueda avance se utiliza para elevar el centro de gravedad de la masa que se apoya sobre ella y parte se disipa en la deformación de la estructura del neumático. En el suelo se provoca una reducción de volumen (compactación) y un desplazamiento por esfuerzo cortante. Siempre se establece un equilibrio entre fuerzas que, las cuales permiten que la rueda avance, o que ésta quede retenida por hundimiento en el suelo. De cualquier modo siempre habrá un equilibrio de fuerzas cuyas resultantes condicionarán el comportamiento de la rueda en el suelo.

Las propiedades físico-mecánicas de los suelos fijan las condiciones para el desplazamiento de los elementos de propulsión y guiado de los vehículos. Hay que tener en cuenta la estructura y granulometría del suelo, la densidad aparente y del "esqueleto" del suelo (densidad de las partículas del suelo), la permeabilidad hidráulica y la humedad en cada momento.

Se han desarrollado diferentes procedimientos para caracterizar los suelos a efectos de traficabilidad de vehículos. Son el "Índice de Cono" (CI) y la "Placa de Carga" métodos que habitualmente se utilizan para predecir el comportamiento de la rueda sobre el suelo.

El Índice de Cono se calcula a partir de la determinación de la fuerza requerida para hacer penetrar en el suelo una varilla provista en el extremo de una punta cónica normalizada (cono de $30^{\circ}$ ) de acuerdo con la norma ASAE S313.2 (ASAE, 1994) que tuvo su origen en las experiencias realizadas para predecir la traficabilidad de los vehículos militares al desplazarse por un suelo natural.

La Placa de Carga permite determinar el hundimiento del suelo para diferentes niveles de presión, lo que puede considerarse equivalente al hundimiento de un propulsor. BEKKER (1969) establece la expresión:

$$
\mathrm{p}=\left[\mathrm{k}_{\mathrm{c}} / \mathrm{b}+\mathrm{k}_{\Phi}\right] \mathrm{z}^{\mathrm{n}}
$$


siendo,

$\mathrm{z}$ - hundimiento de la placa;

b - anchura de la placa cargada;

$\mathrm{k}_{\mathrm{c}}$ - coeficiente que define la cohesión del suelo, $\mathrm{y}$

$\mathrm{k}_{\Phi}$ - coeficiente que define el rozamiento del suelo.

BEKKER (1969) trabaja con presiones expresadas en libras por pulgada cuadrada (psi), y hundimiento y anchura de placa en pulgadas. Las dimensiones de $\mathrm{k}_{\mathrm{c}} \mathrm{y} \mathrm{k}_{\Phi}$ dependen del exponente " $n$ ". Siendo las correspondientes ecuaciones de dimensión, en función de fuerza y longitud, las siguientes:

$$
\mathrm{k}_{\mathrm{c}}=\mathrm{FL}^{-(1+\mathrm{n})} ;\left[\mathrm{k}_{\Phi}\right]=\mathrm{FL}^{-(2+\mathrm{n})}
$$

El hundimiento de una placa en el suelo se puede relacionar directamente con el hundimiento de una rueda de la misma anchura en el suelo, lo que permite deducir la resistencia de la rueda a rodar sobre el suelo. El CI del suelo permite esto mismo, auque no esté tan directamente vinculado al hundimiento del propulsor.

En el caso de que la rueda tenga encomendada la propulsión del vehículo que la monta, el avance depende de la capacidad del suelo para resistir esfuerzo cortante. Para determinar la capacidad del suelo para soportar las cargas horizontales que permiten el avance de la rueda que impulsa a un vehículo se puede utilizar, en una primera aproximación, una placa de cargada con garras clavadas en el suelo, sobre la que se aplica una fuerza horizontal.

La fuerza necesaria para desplazar la placa depende de la carga vertical que gravita sobre ella. La rotura del suelo en el límite de las garras depende de las características del suelo, especialmente de los contenidos de arcilla, limo y arena y de la humedad del mismo. Si se representan gráficamente, en un diagrama las fuerzas de rotura junto con las cargas aplicadas sobre la placa, se obtiene una línea recta, conocida como recta de Coulomb: siendo,

$$
\tau=\mathrm{F} / \mathrm{A}=\mathrm{c}+\mathrm{Q} / \mathrm{A} \operatorname{tg} \varphi=\mathrm{c}+\mathrm{p} \operatorname{tg} \varphi
$$

p - presión de la placa sobre el suelo;

c - cohesión del suelo, y

$\varphi$ - ángulo de rozamiento interno (en grados sexagesimales).

En el desarrollo de la teoría de Coulomb la presión sobre la placa se expresa en $\mathrm{kgf} \mathrm{cm}^{-2}$, por lo que también se podría expresar en pascales $(\mathrm{Pa})$. En este caso, las dimensiones de c y de $\mathrm{p} \operatorname{tg} \varphi$ vendrían dadas en $\mathrm{Pa}$.

Esto se puede tomar como una referencia para fijar el límite de carga horizontal que puede aplicar una rueda o cadena en el suelo, aunque los valores obtenidos no se deben utilizarse como indicadores, ya que no reflejan los que corresponden a una buena eficiencia energética en la propulsión.

La propulsión del vehículo solo se produce cuando el esfuerzo cortante que ejerce la rueda no supera el límite de resistencia del suelo, pero en cualquier caso hay una pérdida de recorrido, que crece a medida que los esfuerzos horizontales aumentan en la misma carga vertical.

Esta pérdida de recorrido, que reduce la distancia real de avance de la rueda en cada vuelta a algo menos de la longitud del perímetro de rodadura, se cuantifica utilizando lo que se conoce como patinamiento o deslizamiento. El coeficiente de deslizamiento se define matemáticamente como la relación:

siendo,

$$
\delta=\left(v_{t}-v_{r}\right) / v_{t}
$$


$\mathrm{v}_{\mathrm{r}}$ - velocidad real de avance, $\mathrm{m} \mathrm{s}^{-1}$;

$\mathrm{v}_{\mathrm{t}}$ - velocidad teórica a la que debería avanzar $=\mathrm{r}_{1} \omega_{1}\left(\mathrm{~m} \mathrm{~s}^{-1}\right)$;

$\mathrm{r}_{1}$ - radio dinámico de de la rueda, $\mathrm{m}, \mathrm{y}$

$\omega_{1}$ - velocidad angular la rueda, $\mathrm{rad} \mathrm{s}^{-1}$.

Por convenio se toma como referencia de velocidad teórica (patinamiento igual a cero) la que se produciría en la rueda autopropulsada circulando sobre suelo firme.

Cuando en un mismo vehículo son motrices las ruedas de ambos ejes, y especialmente en le caso de tractores de doble tracción asistida (FWD), la diferencia de velocidades periféricas en las ruedas del eje delantero con respecto al trasero induce comportamientos anómalos que reducen la capacidad de tracción, como el "power hop".

En los trabajos experimentales realizados los departamentos de ingeniería de distintos fabricantes, como los de BRIXIUS \& ZOZ (1975), WISMER \& LUTH (1971) en la empresa Deere \& Co, y otros centros de investigación (NIAE, WES, etc.), son una base para predecir el comportamiento del neumático en suelo.

Así, a partir de los valores experimentales, se han establecido unos coeficientes de resistencia a la rodadura (Tabla 1 ).

TABLA 1. Valores del coeficiente de resistencia a la rodadura en diferentes tipos de suelo.

Resistance coefficient values for rolling in different types of soil.

\begin{tabular}{lccc}
\hline $\begin{array}{c}\text { Naturaleza y Estado del } \\
\text { Suelo }\end{array}$ & $\begin{array}{c}\text { Coeficiente de Resistencia } \\
\text { a la Rodadura }(\mathrm{k})^{1}\end{array}$ & $\begin{array}{c}\text { Índice de Cono } \\
(\mathrm{CI})[\mathrm{kPa}]\end{array}$ & $\begin{array}{c}\text { Número } \\
\text { Característico }\left(\mathrm{B}_{\mathrm{n}}\right)\end{array}$ \\
\hline Carretera en buen estado & 0,02 a 0,04 & - & \\
Camino de tierra afirmado & 0,03 a 0,05 & - & \\
Camino de tierra & 0,04 a 0,06 & - & 80 \\
Suelo baldío & 0,06 a 0,10 & 1.800 & 55 \\
Rastrojo seco & 0,08 a 0,10 & 1.200 & 40 \\
Tierra labrada & 0,10 a 0,20 & 900 & 20 \\
Arena y suelo muy suelto & 0,15 a 0,30 & $450-250$ & \\
${ }^{1}$ La resistencia a la rodadura se obtiene multiplicando el peso del vehículo por el valor del coeficiente del suelo por el \\
que se circula.
\end{tabular}

Utilizando como referencia el CI del suelo, sobre la base de las presiones de inflado habituales en los neumáticos de los tractores agrícolas y considerando que su deflexión es del 20\%, se puede calcular el "número característico de la rueda" $\left(\mathrm{B}_{\mathrm{n}}\right)$, número adimensional definido en la norma ASAE D497.4 (ASAE, 1999) (en unidades métricas). siendo,

Siendo: $\quad \mathrm{B}_{\mathrm{n}}=\left(\mathrm{CI} \mathrm{b} d / \mathrm{P}_{1,2}\right)[1+5(\Delta / \mathrm{h})] /[1+3(\mathrm{~b} / \mathrm{d})]$

CI - índice de cono del suelo $(\mathrm{kPa})$, según ASAE S313;

$\mathrm{P}_{1,2}$ - carga dinámica sobre la rueda, $\mathrm{kN}$;

$\mathrm{b}$ - anchura del balón de la rueda, $\mathrm{m}$;

$\mathrm{d}$ - diámetro de la rueda cargada, $\mathrm{m}$;

$\mathrm{h}$ - altura del flanco del neumático, $\mathrm{m}$, y

$\Delta$ - deflexión del neumático en las condiciones de carga, $\mathrm{m}$.

A partir del número característico de movilidad de la rueda $\left(\mathrm{B}_{\mathrm{n}}\right)$, se calcula el coeficiente de resistencia a la rodadura por la expresión:

siendo,

$\mathrm{k}=\mathrm{R}_{\mathrm{k}} / \mathrm{P}_{1,2}=\left(1,0 / \mathrm{B}_{\mathrm{n}}\right)+0,04+\left(0,5 \delta / \sqrt{ } \mathrm{B}_{\mathrm{n}}\right)$

$\mathrm{R}_{\mathrm{k}}$ - resistencia a la rodadura, $\mathrm{kN}$;

$\mathrm{P}_{1,2}$ - carga dinámica sobre la rueda, $\mathrm{kN}, \mathrm{y}$

$\delta$ - deslizamiento de la rueda, decimal. 
Respecto a la relación entre el neumático y la capacidad de tracción, es el coeficiente de adherencia el que refleja el comportamiento del neumático. Para el cálculo del empuje que puede proporcionar el neumático motriz (coeficiente de adherencia), según ASAE (1999), también en unidades métricas, se utiliza la expresión:

siendo,

$$
\mu=E_{1} / P_{1}=0,88\left(1-e^{-0.1 B n}\right)\left(1-e^{-0.7 .5 \delta}\right)-1 / B_{n}-\left(0,5 \delta / \sqrt{ } B_{n}\right)
$$

$\mu$ - coeficiente de adherencia;

$\mathrm{E}_{1}$ - empuje de la rueda, $\mathrm{kN}$;

$\mathrm{P}_{1}$ - carga dinámica sobre la rueda, $\mathrm{kN}$;

e - base del logaritmo natural, y

$\delta$ - deslizamiento de la rueda, decimal.

La eficiencia en tracción de la rueda motriz (TE) será:

$\mathrm{TE}=(1-\delta)\left(\mu-\mathrm{R}_{\mathrm{k}}\right) / \mu$ siendo,

$\delta$ - deslizamiento de la rueda, $\%$;

$\mathrm{R}_{\mathrm{k}}$ - resistencia a la rodadura, $\mathrm{kN}, \mathrm{y}$

$\mu$ - coeficiente de adherencia.

Las mayores eficiencias se consiguen cuando el patinamiento toma valores próximos a los indicados en la tabla 2.

TABLA 2. Patinamiento recomendado para conseguir la máxima eficiencia en función del tipo de suelo. Sliding is recommended in order to achieve maximum efficiency upon type of soil.

Tipo de Suelo Patinamiento Óptimo (\%) Eficiencia en Tracción

$\begin{array}{ccc}\text { Firme } & 4-8 & 0,93 \\ & 8-10 & 0,78 \\ \text { Blando } & 11-13 & 0,64 \\ & 14-16 & 0,52\end{array}$

Al crecer los valores de deslizamiento, la eficiencia se reduce de manera significativa. Hay limitaciones en el intervalo para el que son suficientemente aproximadas estas fórmulas de predicción.

Se puede reducir la presión de contacto rueda/suelo para mejorar la eficiencia en tracción utilizando neumáticos de diferente dimensión o con sistema de construcción distinto. En el caso de los neumáticos radiales, el aumento de la superficie de contacto hace que mejore la eficiencia y la capacidad e tracción.

Además, hay que considerar que el relieve del neumático es uno de los elementos esenciales para su selección y a su diseño, el fabricante dedica gran parte de su investigación. El número total de garras, la altura de estas y el ángulo de las mismas con respecto al plano medio del tractor son parámetros que se deben considerar, en especial estas dos últimas, ya que presentan una mayor variabilidad.

Un neumático con más altura de garra trabajando en suelo agrícola tiene un rendimiento menor, salvo si realiza un gran esfuerzo de tracción. Esto lleva a aconsejar, como regla práctica, que no se debe utilizar un taco alto más que cuando el terreno es excesivamente blando. En cualquier caso el neumático que mejor se comporta para unas determinadas condiciones es aquel que puede clavar completamente la garra en el suelo por donde realiza una labor.

Mayores diferencias aparecen en lo que respecta al ángulo de la garra con el plano medio del tractor. El ángulo de las garras es un compromiso entre la capacidad de agarre y la limpieza del 
intervalo entre dos garras consecutivas. En condiciones de clima húmedo, o cuando se deben trabajar suelos húmedos, se obtienen mejores resultados con ángulo de garras de $45^{\circ}$ con el plano medio del neumático. En neumáticos para suelos más secos el ángulo de $67^{\circ}\left(23^{\circ}\right.$ con un plano perpendicular al avance) proporciona limpieza suficiente y aumenta la capacidad de tracción.

\section{CRITERIOS PARA DIMENSIONAMIENTO DE LOS NEUMÁTICOS}

Si se analizan con detenimiento las características esenciales de los tractores que compiten en un segmento de potencia, se puede observar que, además de tener motores equivalentes, los neumáticos también presentan gran similitud.

El adecuado dimensionamiento de los neumáticos puede tener una importancia decisiva, ya que unos neumáticos subdimensionados impiden convertir la potencia del motor en potencia de tracción, especialmente en operaciones lentas que demandan una elevada tracción, aunque también actúan como dispositivo de seguridad que permite proteger las transmisiones cuando el motor del tractor les suministra un par más elevado del que estas pueden soportar, sobre todo en la parte final del recorrido del motor a las ruedas.

Tampoco la utilización de neumáticos sobredimensionados es una buena solución, ya que la superficie de apoyo del neumático es una consecuencia de la carga sobre la rueda y la presión de inflado, o lo que es lo mismo, la superficie de contacto con el suelo es menor, ya que una parte de la banda de rodadura no toca el suelo.

La relación que existe entre la fuerza tangencial que llega a cada una de las ruedas motrices, con la potencia disponible en el motor para una determinada velocidad de avance y la capacidad del suelo para resistir el esfuerzo cortante, en función de la carga vertical que gravite sobre la rueda, permite calcular la masa del tractor y dimensionar las ruedas neumáticas a partir de la potencia del motor.

\section{Capacidad de carga del neumático}

La capacidad de carga de un neumático está relacionada con sus dimensiones y con la presión de inflado. En el marcado de un neumático, además del que permite conocer sus dimensiones principales, como el ancho y el diámetro de la llanta, aparece una indicción sobre la capacidad de carga, denominado "índice de carga" (IC).

Este índice se ha normalizado y se ha establecido a partir de las capacidades de carga acordadas por diferentes instituciones de normalización, mediante escalones que aumentan con la capacidad de carga. La relación entre la carga que puede soportar el neumático y el índice correspondiente se presenta en la figura 1.

Estos valores de capacidad de carga corresponden a la rueda inflada a una presión de referencia de $160 \mathrm{kPa}$ (en los manuales y recomendaciones de ETRTO (2001) las presiones de inflado se expresan en bar; $160 \mathrm{kPa}=1,6$ bar) que es la que se ha establecido para los neumáticos de uso agrícola por entenderse que es un valor límite para las ruedas que han de trabajar en el campo. También se relaciona con la velocidad máxima admitida, que se toma generalmente como $40 \mathrm{~km} \mathrm{~h}^{-1}$ (marcado de velocidad A8).

Sin embrago, al trabajar en un suelo agrícola conviene reducir la presión de inflado, limitando así la compactación del suelo. También, una rueda a menor presión de inflado que la de referencia reduce su capacidad de carga.

El neumático elegido debe disponer de una capacidad de carga suficiente para soportar la que se deriva de la masa del tractor y de las cargas dinámicas que aparecen con el trabajo (masas y reacciones de los implementos). Hay que tener en cuenta que las cargas máximas de referencia, indicadas en los neumáticos, son superiores a las que se pueden aplicar en el suelo agrícola, dado que reflejan la resistencia del neumático a una elevada presión de inflado, solo admisible cuando se circula sobre pavimento. 
Correspondencias entre indices de carga (IC) y capacidad de carga por rueda (kg)

\begin{tabular}{|cccccccc|}
\multicolumn{6}{c}{ Correspondencias entre indices de carga (IC) y capacidad de carga por rueda (kg) } \\
\hline IC & $\mathrm{kg}$ & $\mathrm{IC}$ & $\mathrm{kg}$ & $\mathrm{IC}$ & $\mathrm{kg}$ & $\mathrm{IC}$ & $\mathrm{kg}$ \\
\hline 60 & 250 & 93 & 650 & 126 & 1700 & 159 & 4375 \\
\hline 61 & 257 & 94 & 670 & 127 & 1750 & 160 & 4500 \\
\hline 62 & 265 & 95 & 690 & 128 & 1800 & 161 & 4625 \\
\hline 63 & 272 & 96 & 710 & 129 & 1850 & 162 & 4750 \\
\hline 64 & 280 & 97 & 730 & 130 & 1900 & 163 & 4875 \\
\hline 65 & 290 & 98 & 750 & 131 & 1950 & 164 & 5000 \\
\hline 66 & 300 & 99 & 775 & 132 & 2000 & 165 & 5150 \\
\hline 67 & 307 & 100 & 800 & 133 & 2060 & 166 & 5300 \\
\hline 68 & 315 & 101 & 825 & 134 & 2120 & 167 & 5450 \\
\hline 69 & 325 & 102 & 850 & 135 & 2180 & 168 & 5600 \\
\hline 70 & 335 & 103 & 875 & 136 & 2240 & 169 & 5800 \\
\hline 71 & 345 & 104 & 900 & 137 & 2300 & 170 & 6000 \\
\hline 72 & 355 & 105 & 925 & 138 & 2360 & 171 & 6150 \\
\hline 73 & 365 & 106 & 950 & 139 & 2430 & 172 & 6300 \\
\hline 74 & 375 & 107 & 975 & 140 & 2500 & 173 & 6500 \\
\hline 75 & 387 & 108 & 1000 & 141 & 2575 & 174 & 6700 \\
\hline 76 & 400 & 109 & 1030 & 142 & 2650 & 175 & 6900 \\
\hline 77 & 412 & 110 & 1060 & 143 & 2725 & 176 & 7100 \\
\hline 78 & 425 & 111 & 1090 & 144 & 2800 & 177 & 7300 \\
\hline 79 & 437 & 112 & 1120 & 145 & 2900 & 178 & 7500 \\
\hline 80 & 450 & 113 & 1150 & 146 & 3000 & 179 & 7750 \\
\hline 81 & 462 & 114 & 1180 & 147 & 3075 & 180 & 8000 \\
\hline 82 & 475 & 115 & 1215 & 148 & 3150 & 181 & 8250 \\
\hline 83 & 487 & 116 & 1250 & 149 & 3250 & 182 & 8500 \\
\hline 84 & 500 & 117 & 1285 & 150 & 3350 & 183 & 8750 \\
\hline 85 & 515 & 118 & 1320 & 151 & 3450 & 184 & 9000 \\
\hline 86 & 530 & 119 & 1360 & 152 & 3550 & 185 & 9250 \\
\hline 87 & 545 & 120 & 1400 & 153 & 3650 & 186 & 9500 \\
\hline 88 & 560 & 121 & 1450 & 154 & 3750 & 187 & 9750 \\
\hline 89 & 580 & 122 & 1500 & 155 & 3875 & 188 & 10000 \\
\hline 90 & 600 & 123 & 1550 & 156 & 4000 & 189 & 10300 \\
\hline 91 & 615 & 124 & 1600 & 157 & 4125 & 190 & \\
\hline 92 & 630 & 125 & 1650 & 158 & 4250 & 191 & \\
\hline & & & & & & & \\
\hline
\end{tabular}

FIGURA 1. Índices de carga de los neumáticos de uso agrícola. (Fuente: ETRTO, 2001). Load indexes for tires used in agricultural purposes. (Source: ETRTO, 2001).

En una primera aproximación se puede establecer que las cargas máximas que recibirán las ruedas de cada eje durante la utilización, en función del tipo de tractor considerado, como se indica en la Tabla 3. La capacidad total de carga del conjunto de los neumáticos debe de ser un $30 \%$ mayor que la de referencia del tractor, como consecuencia de que durante el trabajo hay unas transferencias de carga variables que deben de soportar los neumáticos de cada eje.

TABLA 3. Masa en cada eje en porcentaje sobre la masa de referencia del tractor (sin implementos). Weight of each axle in percentage over reference weight of tractor (without tools).

\begin{tabular}{lcc}
\hline Tipo & Delanteras & Traseras \\
\hline Simple tracción (2WD) & $30 \%$ & $100 \%$ \\
Doble tracción (FWA) & $50 \%$ & $80 \%$ \\
Doble tracción (4WA) & $70 \%$ & $60 \%$ \\
\hline
\end{tabular}

Por otra parte, la masa de referencia se puede obtener añadiendo lastres en función del tipo de trabajo que se realiza, para aprovechar eficientemente la potencia disponible (minimizando pérdidas por rodadura y patinamiento)

La masa necesaria de un tractor para trabajos de tracción en función de su potencia se puede calcular con la siguiente expresión matemática:

siendo,

$$
\mathrm{P}=\mathrm{N} \quad 0,75 \eta_{\mathrm{t}} 270 /\left(\mathrm{v}_{\mathrm{t}} \mu \mathrm{c}_{\mathrm{TR}}\right)
$$

$\mathrm{P}$ - masa necesaria (incluido lastre), $\mathrm{kg}$;

$\mathrm{N}$ - potencia del motor (en cv) utilizada (se considera que se utiliza el 75\% de la máxima);

$\eta_{\mathrm{t}}$ - eficiencia en la transmisión ( $90 \%$; equivalente a $10 \%$ de pérdidas);

$\mathrm{v}_{\mathrm{t}}$ - velocidad teórica de avance en $\mathrm{km} \mathrm{h}^{-1}$ ( $\sin$ descontar resbalamiento);

$\mu$ - coeficiente de adherencia (tipo de suelo: 0,5 a 0,9), y

$\mathrm{c}_{\mathrm{TR}}$ - coeficiente de tipo de tractor (1,00 en tractores del tipo 4WD y FWA; 0,85 en tractores 2WD) 
Aplicando esta expresión matemática a diferentes tractores con potencias crecientes, las masas de referencia necesarias para cada caso aparecen calculadas en la Tabla 4.

TABLA 4. Masa total necesaria en el tractor $(\mathrm{kg})$ en función de la potencia del motor que se va a utilizar en trabajos de tracción, para diferentes velocidades de trabajo sobre rastrojo $(\mu=0,6)$. Necessary tractor total weight upon engine power used in traction work, for different work velocities over stubble $(\mu=0,6)$.

\begin{tabular}{ccccccc}
\hline Velocidad & 4,5 & $\mathrm{~km} \mathrm{~h}^{-1}$ & 6,5 & $\mathrm{~km} \mathrm{~h}^{-1}$ & 8,5 & $\mathrm{~km} \mathrm{~h}^{-1}$ \\
\hline Coef. TR & $2 \mathrm{WD}$ & FWA & $2 \mathrm{WD}$ & $\mathrm{FWA}$ & $2 \mathrm{WD}$ & $\mathrm{FWA}$ \\
\hline Potencia (cv) & 0,85 & 1,00 & 0,85 & 1,00 & 0,85 & 1,00 \\
\hline 50 & & & & & & \\
60 & 3.971 & 3.375 & 2.749 & 2.337 & 2.102 & 1.787 \\
70 & 4.765 & 4.050 & 3.299 & 2.804 & 2.522 & 2.144 \\
80 & 5.559 & 4.725 & 3.848 & 3.271 & 2.943 & 2.501 \\
90 & 6.353 & 5.400 & 4.398 & 3.738 & 3.363 & 2.859 \\
100 & 7.147 & 6.075 & 4.948 & 4.206 & 3.784 & 3.216 \\
110 & 7.941 & 6.750 & 5.498 & 4.673 & 4.204 & 3.574 \\
120 & & 7.425 & 6.048 & 5.140 & 4.625 & 3.931 \\
130 & & 8.100 & 6.597 & 5.608 & 5.045 & 4.288 \\
140 & & 8.775 & 7.147 & 6.075 & 5.465 & 4.646 \\
150 & & 9.450 & 7.697 & 6.542 & 5.886 & 5.003 \\
160 & & 10.125 & & 7.010 & 6.306 & 5.360 \\
170 & & 10.800 & & 7.477 & 6.727 & 5.718 \\
180 & & 11.475 & & 7.944 & 7.147 & 6.075 \\
190 & & 12.150 & & 8.412 & 7.567 & 6.432 \\
200 & 12.825 & & 8.879 & 7.988 & 6.790 \\
\hline
\end{tabular}

Notas: La masa puede ser del propio tractor o inducida por los implementos utilizados, pero en ambos casos sirve de referencia para el dimensionamiento de los neumáticos.

Las velocidades utilizadas son teóricas, y no tienen en cuenta el patinamiento.

Se considera que la potencia utilizada es el $75 \%$ de la potencia máxima disponible en el motor

Esto significa que en un tractor 4WD con $100 \mathrm{cv}$ de potencia, los neumáticos deberían soportar, en su conjunto, una masa de $6.750 \mathrm{~kg}$, que, según lo indicado en la Tabla 4, hace aconsejable elegir neumáticos delanteros y traseros con las siguientes capacidades de carga:

\begin{tabular}{|c|c|c|}
\hline Neumáticos & Total $(\mathrm{kg})$ & Por rueda $(\mathrm{kg})$ \\
\hline$\overline{\text { Delanteros }}$ & $6.75050 / 100=3.375$ & $3.375 / 2=1.688$ \\
\hline Traseros & $6.75080 / 100=5.400$ & $5.400 / 2=2.700$ \\
\hline
\end{tabular}

A partir de estos valores se puede elegir el índice de carga del neumático, pero hay que establecer previamente las presiones de inflado aconsejables para el trabajo de campo, ya que los valores de carga admisible a los que se refiere el índice de carga del neumático son para presiones de inflado de $160 \mathrm{kPa}$.

\section{Variación de la capacidad de carga con la presión de inflado}

El diseño de cualquier neumático se realiza de manera que su estructura alcance una forma determinada cuando la carga que gravita sobre él, se equilibra con la presión de inflado. Si se reduce la carga sobre la rueda hay que bajar la presión de inflado, para que la superficie de apoyo se mantenga constante, ya que en caso contrario se reduce la capacidad de tracción del neumático, a la vez que incrementa su desgaste. 


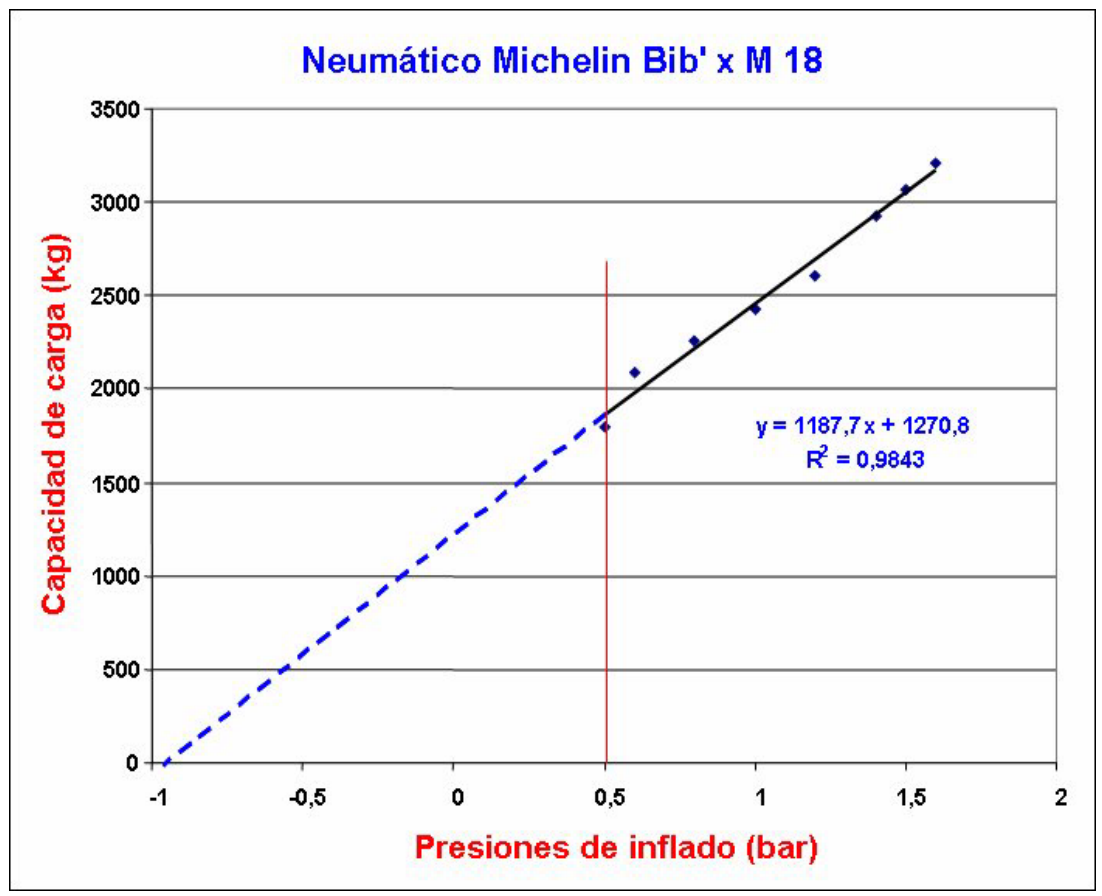

FIGURA 2. Variación de la capacidad de carga en función de la presión de inflado. Load capacity range upon inflation pressure.

Por el mismo criterio, un aumento de la carga resulta posible incrementando la presión de inflado, aunque esto puede afectar la resistencia del neumático y reducir su vida útil, salvo que esta sobrecarga se produzca con una velocidad de desplazamiento inferior al código de velocidad del neumático. Esto se aplica a la elección de los neumáticos del eje delantero cuando se utiliza palas frontales, o máquinas que producen un efecto similar.

En los catálogos de los fabricantes de neumáticos, en ocasiones se encuentran tablas que relacionan la presión de inflado con la capacidad de carga del neumático. En otros casos solo se indica la capacidad de carga máxima (para la velocidad admitida como referencia) y la presión de inflado correspondiente, por lo que habrá que calcular la presión de inflado más apropiada cuando la carga real sobre la rueda esté por debajo de la carga de referencia.

A este respecto, se puede observar que la relación entre la capacidad de carga y la presión de inflado se mantiene lineal (Figura 2), por lo que resulta sencillo el cálculo de la presión de inflado a partir de referencia de carga nominal del neumático.

En consecuencia, si se parte de un neumático con una capacidad de carga de $3.000 \mathrm{~kg}$ a $160 \mathrm{kPa}(1,6$ bar) la capacidad de carga para la presión de inflado de 1,1 bar será:

$$
\mathrm{C}_{1.1}=3000(1,1+1,0) /(1,6+1,0)=2.423 \mathrm{~kg}
$$

Lo que se puede expresar de manera genérica como: siendo:

$$
\mathrm{C}_{\mathrm{pi}}=\mathrm{C}_{\max }\left(\mathrm{p}_{\mathrm{i}}+1\right) /\left(\mathrm{p}_{\max }+1\right)
$$

$\mathrm{C}_{\mathrm{pi}}$ - capacidad de carga $(\mathrm{kg})$ a la presión de inflado, $\mathrm{p}_{\mathrm{i}}$;

$\mathrm{C}_{\max }$ - capacidad de carga $(\mathrm{kg})$ a la presión de inflado de referencia;

$\mathrm{p}_{\mathrm{i}}$ - presión de inflado utilizada, $\mathrm{kPa}, \mathrm{y}$

$\mathrm{p}_{\max }$ - presión de inflado de referencia $(160 \mathrm{kPa}=1,6 \mathrm{bar})$.

Hay que tener en cuanta que si la presión de inflado es inferior a un determinado valor, dependiente del tipo de neumático y de las características de la llanta utilizada, el neumático tiende a girar en la llanta, por lo que los fabricantes limitan la presión mínima de inflado. 


\section{APLICACIÓN PARA TRACTORES DE TRACCIÓN DELANTERA ASISTIDA (FWA)}

Se estima que un tractor con tracción delantera asistida debe de disponer de neumáticos con una capacidad de carga igual al $130 \%$ de la masa necesaria para poder convertir un determinado porcentaje de la potencia de su motor en potencia de tracción, para velocidades de trabajo habituales de las operaciones agrícolas.

De esta masa total, el $80 \%$ estaría sobre las ruedas del eje trasero y el $50 \%$ sobre las del eje delantero.

En consecuencia, contando con dos ruedas traseras de $3.000 \mathrm{~kg}$ de capacidad de carga nominal, que se convierten en $2.310 \mathrm{~kg}$ a una presión de inflado de $100 \mathrm{kPa}$ (1,0 bar) la masa del tractor que monte estas ruedas en el eje trasero podría ser:

$$
2310 \times 2 / 0,8=5.775 \mathrm{~kg}
$$

\section{Potencia de tracción}

Suponiendo que se ha lastrado el tractor hasta conseguir esta masa, bien mediante contrapesos o mediante las fuerzas que pueden transferir los aperos durante el trabajo, la fuerza de tracción que puede proporcionar el tractor en un suelo agrícola con coeficiente de adherencia (al tratarse de un tractor de doble tracción) será:

$$
5775 \text { x } 0,6=3.465 \mathrm{~kg}
$$

Dado que potencia de tracción es igual a la fuerza por la velocidad real de avance, la potencia de tracción será:

$$
\mathrm{N}(\mathrm{cv})=3.465(\mathrm{~kg}) \times \text { velocidad }\left(\mathrm{km} \mathrm{h}^{-1}\right) / 270
$$

Siendo el 270 el factor para relacionar estas magnitudes utilizando las unidades de medida del Sistema Técnico. La unidad cv pertenece al sistema técnico; si se expresara la potencia de los tractores en $\mathrm{kW}$, el factor 270 se cambiaría por 360 y el peso del tractor se expresaría en daN.

Si consideramos como velocidad de referencia, para trabajos agrícolas en los que la demanda de potencia de tracción es elevada, la de $6,5 \mathrm{~km} \mathrm{~h}^{-1}$ (velocidad real), la potencia de tracción del tractor deberá de ser:

$$
\mathrm{N}(\mathrm{cv})=3.465(\mathrm{~kg}) \times 6,5\left(\mathrm{~km} \mathrm{~h}^{-1}\right) / 270=83 \mathrm{cv}
$$

\section{Potencia en el motor}

Para que un tractor con los neumáticos indicados, lastrado de acuerdo con las posibilidades de los mismos y trabajando en las condiciones anteriormente fijadas, pueda suministrar esta potencia neta en tracción, necesita una potencia mayor en el motor.

Suponiendo que se trabaja en un suelo con rastrojo, patinamiento entre 9 y $12 \%$, la eficiencia en tracción, considerada como a relación entre la potencia de tracción y la potencia del motor, puede ser del $73 \%$, por lo que, para el ejemplo del apartado anterior, con una potencia de tiro de $83 \mathrm{cv}$ se necesitaría, al menos, $83 / 0,73=114$ potencia de motor.

Sin embargo, no es conveniente que el motor del tractor trabaje de manera continua a su potencia máxima, por lo que conviene mayoral el valor calculado de manera que sea el $75-80 \%$ de la potencia máxima disponible. De esta manera se aprovecha la inversión realizada, a la vez que se mantiene bajo el consumo de combustible. En consecuencia, estos neumáticos serían apropiados para un tractor entre 140 y $150 \mathrm{cv}$ de potencia en el motor.

\section{Con neumáticos radiales de serie normal}

A partir de la información disponible en el manual de neumáticos de ERTRO (2001) se han seleccionado los neumáticos radiales de serie normal con radio índice entre 600 y $925 \mathrm{~mm}$, lo que se corresponde con circunferencias de rodadura teóricas (a efectos de cálculo de la velocidad máxima de circulación) entre 3.770 y $5.812 \mathrm{~mm}$.

Se han analizado de manera separada, agrupándolos por la sección del neumático, de manera que el índice de carga correspondiente (manual ERTRO con presión de inflado de 1,6 bar) irá aumentando a medida que lo hace el diámetro de la llanta. 
Aplicando la metodología anteriormente desarrollada, se puede estimar la masa adecuada para el tractor (en trabajos de tracción que demandan elevada potencia a velocidad real de $6,5 \mathrm{~km} \mathrm{~h}^{-1}$ ) que monta dichos neumáticos traseros, y los valores de potencia de tracción y potencia mínima del motor que lo hace posible.

En la Tabla 5 se presentan los valores calculados, que se representan gráficamente en la Figura 3 , para las distintas secciones de neumático y con cada dimensión de llanta en pulgadas, según aparece en el marcado del neumático radial de serie normal. Los valores de potencia mínima indicados hay que incrementarlos, como ya se ha indicado, entre un 20 - 25\%, si se quiere aprovechar adecuadamente la potencia que se compra manteniendo un bajo consumo de combustible.

TABLA 5. Masa del tractor y potencia mínima de su motor en función de las dimensiones de sus neumáticos traseros (radiales serie normal). Hipótesis: presión de inflado de 1,0 bar; coeficiente reductor 0,77; carga de referencia sobre el eje trasero: 0,8 de la masa del tractor; coeficiente de adherencia: 0,6; velocidad real: $6,5 \mathrm{~km} \mathrm{~h}^{-1}$; eficiencia en tracción: 0,73 (deslizamiento $(9-12 \%)$.

Tractor weight and engine minimum power upon dimensions of rear tires (normal radial series). Hypothesis: inflation pressure of 1.0 bar; reducing coefficient 0.77 ; reference load over rear axle: 0.8 of tractor weight; adherence coefficient: 0.6 ; actual velocity: $6.5 \mathrm{~km} \mathrm{~h}^{-1}$; traction efficiency: 0.73 (glide: 9-12\%).

\begin{tabular}{|c|c|c|c|c|c|c|c|c|c|c|c|c|}
\hline & \multicolumn{6}{|c|}{$14,9(378 \mathrm{~mm})$} & \multicolumn{6}{|c|}{$16,9(429 \mathrm{~mm})$} \\
\hline $\begin{array}{l}\text { Radio } \\
\text { Índice }\end{array}$ & Llanta & $\begin{array}{c}\mathrm{IC} \\
1,6 \mathrm{bar}\end{array}$ & \begin{tabular}{|c|}
$\begin{array}{c}\text { Rueda } \\
(\mathrm{kg})\end{array}$ \\
\end{tabular} & $\begin{array}{c}\text { Masa } \\
(\mathrm{kg})\end{array}$ & $\begin{array}{l}\text { Pot_Tr } \\
\text { (cv) }\end{array}$ & $\begin{array}{c}\text { Pot_min } \\
\text { (cv) }\end{array}$ & Llanta & $\begin{array}{c}\mathrm{IC} \\
1,6 \mathrm{bar}\end{array}$ & $\begin{array}{c}\text { Rueda } \\
(\mathrm{kg})\end{array}$ & $\begin{array}{c}\text { Masa } \\
(\mathrm{kg})\end{array}$ & $\begin{array}{l}\text { Pot_Tr } \\
\text { (cv) }\end{array}$ & $\begin{array}{c}\text { Pot_min } \\
\text { (cv) }\end{array}$ \\
\hline 600 & R24 & 126 & 1.700 & 3.273 & 47 & 65 & & & & & & \\
\hline 625 & R26 & 127 & 1.750 & 3.369 & 49 & 67 & R24 & 134 & 2.120 & 4.081 & 59 & 81 \\
\hline 650 & R28 & 128 & 1.800 & 3.465 & 50 & 69 & R26 & 135 & 2.180 & 4.197 & 61 & 83 \\
\hline 675 & R30 & 129 & 1.850 & 3.561 & 51 & 70 & R28 & 136 & 2.240 & 4.312 & 62 & 85 \\
\hline 700 & & & & & & & R30 & 137 & 2.300 & 4.428 & 64 & 88 \\
\hline 725 & & & & & & & & & & & & \\
\hline 750 & & & & & & & R34 & 139 & 2.430 & 4.678 & 68 & 93 \\
\hline 775 & R38 & 133 & 2.060 & 3.966 & 57 & 78 & & & & & & \\
\hline 800 & & & & & & & R38 & 141 & 2.575 & 4.957 & 72 & 98 \\
\hline 825 & & & & & & & R42 & 143 & 2.745 & 5.284 & 76 & 105 \\
\hline 850 & & & & & & & & & & & & \\
\hline 875 & & & & & & & & & & & & \\
\hline 900 & & & & & & & & & & & & \\
\hline 925 & & & & & & & & & & & & \\
\hline 950 & & & & & & & & & & & & \\
\hline
\end{tabular}

\begin{tabular}{|c|c|c|c|c|c|c|c|c|c|c|c|c|}
\hline & \multicolumn{6}{|c|}{$18,4(467 \mathrm{~mm})$} & \multicolumn{6}{|c|}{$20,8(528 \mathrm{~mm})$} \\
\hline $\begin{array}{l}\text { Radio } \\
\text { Índice }\end{array}$ & Llanta & \begin{tabular}{|c|}
$\mathrm{IC}$ \\
$1,6 \mathrm{bar}$
\end{tabular} & $\begin{array}{c}\text { Rueda } \\
\text { (kg) }\end{array}$ & $\begin{array}{c}\text { Masa } \\
(\mathrm{kg})\end{array}$ & $\begin{array}{c}\text { Pot_Tr } \\
\text { (cv) }\end{array}$ & $\begin{array}{c}\text { Pot_min } \\
(\mathrm{cv})\end{array}$ & Llanta & \begin{tabular}{|c|}
$\mathrm{IC}$ \\
$1,6 \mathrm{bar}$
\end{tabular} & $\begin{array}{l}\text { Rueda } \\
\text { (kg) }\end{array}$ & $\begin{array}{l}\text { Masa } \\
(\mathrm{kg})\end{array}$ & $\begin{array}{c}\text { Pot_Tr } \\
\text { (cv) }\end{array}$ & \begin{tabular}{|c|} 
Pot_min \\
(cv)
\end{tabular} \\
\hline \\
\hline \multicolumn{13}{|l|}{625} \\
\hline 650 & R24 & 139 & 2.430 & 4.678 & 68 & 93 & & & & & & \\
\hline 675 & R26 & 140 & 2.550 & 4.909 & 71 & 97 & & & & & & \\
\hline 700 & R28 & 141 & 2.575 & 4.957 & 72 & 98 & & & & & & \\
\hline 725 & R30 & 142 & 2.650 & 5.101 & 74 & 101 & & & & & & \\
\hline \multicolumn{13}{|l|}{750} \\
\hline 775 & R34 & 144 & 2.800 & 5.390 & 78 & 107 & & & & & & \\
\hline \multicolumn{13}{|l|}{800} \\
\hline 825 & R38 & 146 & 3.000 & 5.775 & 83 & 114 & R34 & 151 & 3.450 & 6.641 & 96 & 131 \\
\hline \multicolumn{13}{|l|}{850} \\
\hline 875 & R42 & 148 & 3.150 & 6.064 & 88 & 120 & & & & & & \\
\hline \multirow{3}{*}{$\begin{array}{l}900 \\
925 \\
950\end{array}$} & & & & & & & R38 & 153 & 3.650 & 7.026 & 101 & 139 \\
\hline & & & & & & & R42 & 155 & \begin{tabular}{|l|}
3.875 \\
\end{tabular} & 7.459 & 108 & 148 \\
\hline & & & & & & & & & & & & \\
\hline
\end{tabular}




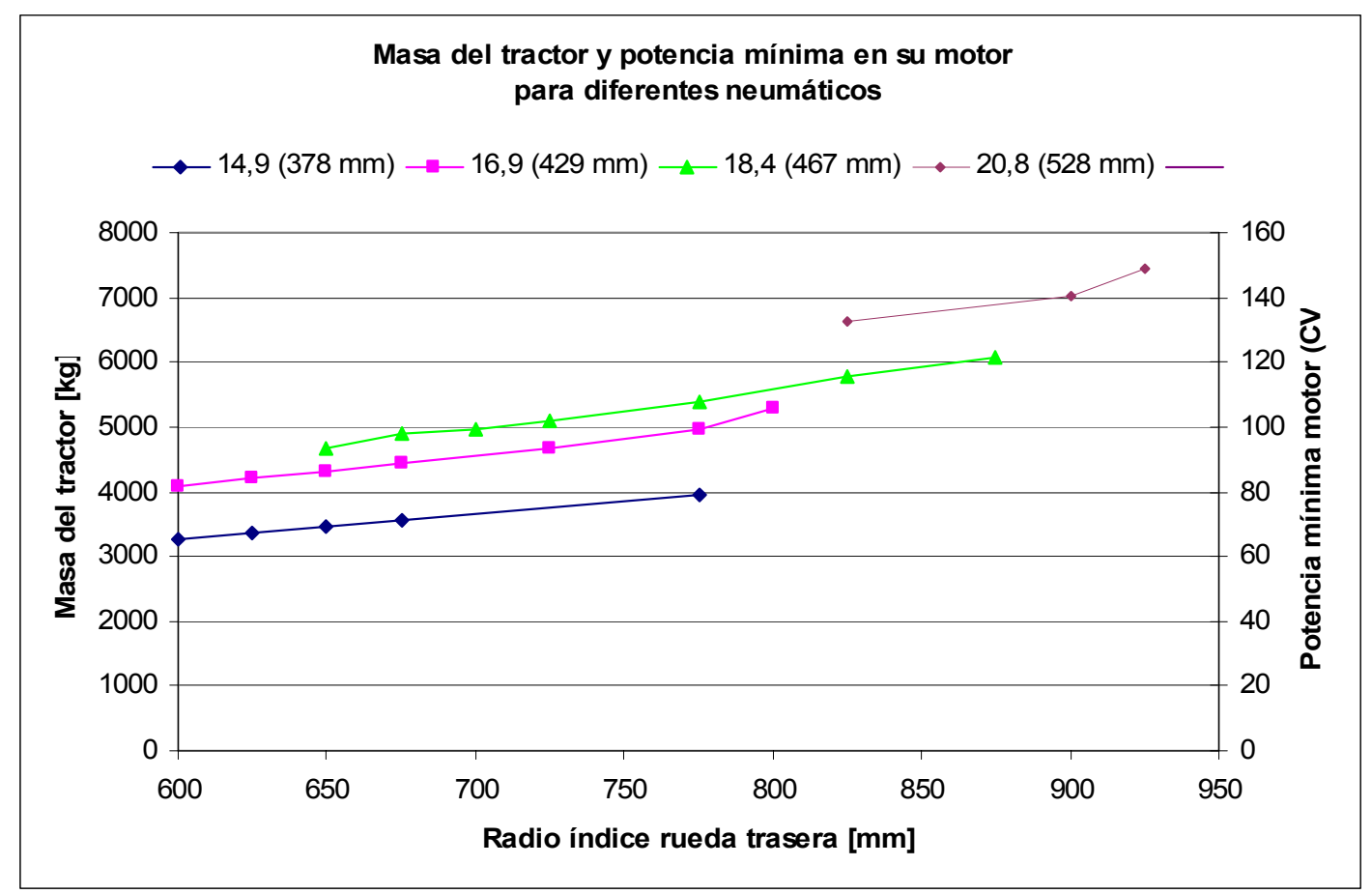

FIGURA 3. Masa del tractor y potencia mínima para diferentes dimensiones de neumáticos. Tractor weight and minimum power for tires different dimension.

Por otra parte, conviene revisar el marcado de los neumáticos delanteros, de manera que el índice de carga correspondiente admita al menos el 50\% de la masa de referencia calculada para el tractor. En ellos puede admitirse una presión de inflado de $160 \mathrm{kPa}(1,6 \mathrm{bar})$, por lo que no sería necesario aplicar un factor de corrección.

El modelo de cálculo utilizado, que queda reflejado en la Tabla 5 permite llegar a una recomendación de tipo práctico: dividiendo por 36 la capacidad de carga nominal de un neumático trasero para un tractor FWA, se conoce la potencia de tracción que podría desarrollar el tractor que las lleva, sobre la base de trabajar a $100 \mathrm{kPa}(1,0$ bar) de presión de inflado y con el adecuado equilibrado del lastre. Para ello, la potencia que se aconseja en el motor puede calcularse dividiendo la capacidad de carga por 21 . Esto significa disponer un motor que supere en un $20 \%$ la potencia mínima necesaria para trabajar en las condiciones establecidas.

\section{Con neumáticos radiales de serie métrica}

Cada vez es más frecuente que el mercado de los neumáticos se realice de una forma diferente, cambiando el primer término (anchura de balón en pulgadas) por dos cifras, la primera de las cuales es la anchura del balón en milímetros y la segunda la relación entre la altura y la anchura del perfil.

Así, un marcado 600/65R38 indicaría que se trata de un neumático de construcción radial, con $600 \mathrm{~mm}$ de anchura del balón y una relación altura anchura igual a 0,65 (del 65\%), para una llanta de 38 pulgadas de diámetro.

Este marcado, que coexiste con el marcado en pulgadas, utiliza como anchuras de de referencia valores de centenas $(400 ; 500, .$.$) y también otros con intervalos más pequeños, como$ $420 ; 440 ; 480 \ldots$, en relaciones de perfil entre 50 y 95, aunque las series que incluyen más modelos son las de perfil $65 ; 70$ y 85 . Está última incluye los modelos que más se aproximan dimensionalmente a los de marcado tradicional, ya que estos mantiene una relación de perfil similar. 
Es frecuente relacionar los neumáticos de marcado métrico con la "baja presión". Esto puede considerarse como válido en algunos casos, ya que se trata de modelos con una gran anchura de balón, pero la presión de inflado es la que marca la presión sobre el suelo, a la vez que se reduce la capacidad de carga; el valor mínimo de presión de inflado que se puede utilizar depende del fabricante considerado, siendo habituales las presiones entre 50 y $70 \mathrm{kPa}$. En cualquier caso la capacidad de carga de referencia corresponde a $160 \mathrm{kPa}$ de presión de inflado (aunque en algunos caso se utiliza como referencia la de $120 \mathrm{kPa}$ ).

En consecuencia, si se considera que se van a utilizar neumáticos traseros inflados durante el trabajo a una presión de $100 \mathrm{kPa}$, todo lo indicado para los neumáticos con marcado en pulgadas resulta válido para los de marcado métrico. Si se desea trabajar a $80 \mathrm{kPa}$, la masa del tractor se reduce en un $10 \%$ con respecto a la calculada con presión de inflado de $100 \mathrm{kPa}$, y por ello la potencia de tracción a $6,5 \mathrm{~km} \mathrm{~h}^{-1}$ de velocidad real de avance sería menor.

El valor de la potencia máxima de tracción a $6,5 \mathrm{~km} \mathrm{~h}^{-1}$ en este caso se puede calcular dividiendo por 40 la capacidad de carga nominal del neumático, y la potencia del motor dividiendo este mismo valor por 23 (en lugar de haber utilizado los valores de 36 y 21, anteriormente indicados)

Lo lógico, si se quiere trabajar con neumáticos inflados a baja presión, sobre la base del tractor con una potencia dada y calculada a partir de neumáticos convencionales, sería actuar al revés, o sea, sobre la base de un neumático con marcado normal de $3.000 \mathrm{~kg}$ de capacidad de carga $(\mathrm{IC}=146)$, se obtendrán, cuando se infla a 1,0 bar, una potencia de tracción de $3.000 / 36=83 \mathrm{cv}$, con una potencia de motor recomendada de $3.000 / 21=143 \mathrm{cv}$. Si se monta un neumático para trabajar a 0.8 bar de presión de inflado su capacidad de carga (a 1,6 bar) debería de ser de $143 \times 23=3.290 \mathrm{~kg}$, o sea IC igual a $149-150$.

En uno y otro caso los neumáticos elegidos deben de tener el mismo radio índice para que no modifiquen la velocidad máxima de circulación, o, mejor, estar incluidos en el mismo grupo RCI (clasificación de los neumáticos por sus circunferencias de rodadura a efectos de compatibilidad de las ruedas delanteras y traseras en tractores de doble tracción)

\section{CONCLUSIONES}

La potencia de tracción que proporciona un tractor de manera eficiente se calcula, de manera bastante aproximada, dividiendo por 36 la capacidad de carga nominal (a $160 \mathrm{kPa}=1,6$ bar) de los neumáticos montados en las ruedas traseras.

En estas condiciones, la potencia del motor que se necesita se calcula dividiendo por 21 este mismo valor de capacidad de carga de los neumáticos traseros. Así se dispone en el motor de una reserva de potencia del $20 \%$ con respecto a la mínima necesaria con una adecuada eficiencia de tracción.

La masa del tractor en trabajos de tracción, sobre la base de $6,5 \mathrm{~km} \mathrm{~h}^{-1}$ de velocidad real, debe de ser igual a la capacidad de carga del neumático trasero multiplicada por 1.925. Así, relación peso potencia del tractor será: $1.925 \times 21=40 \mathrm{~kg} \mathrm{cv}^{-1}$. En estas condiciones el deslizamiento se mantendrá entre el 9 y el $12 \%$.

Si se dispone de un motor de más potencia de la que se deduce aplicando esta metodología, no se podrá utilizar para transformarla en potencia de tracción de manera eficiente a baja velocidad $\left(6,5 \mathrm{~km} \mathrm{~h}^{-1}\right)$ con los neumáticos disponibles. Podría hacerlo trabajando más deprisa, si esto resulta posible (menor esfuerzo de tracción) utilizando la toma de fuerza.

\section{BIBLIOGRAFÍA}

ASAE D497.4. Agricultural machinery management data. In: ASAE Standards. St Joseph, 1999.

ASAE S313.2. Soil cone penetrometer. In: ASAE Standards. St Joseph, 1994. 
BARTHELEMY, P.; BILLOT J-F.; BIDET J-M. Choisir les neumatiques. Paris: ITCF, 1991.

BEKKER, M.G. Introduction to terrain-vehicle system. Ann Arbor: University of Michigan, 1969.

BRIXIUS, W.W.; ZOZ, F.M. Tractor-implement perfomance, 1975. documento de difusión restringida

ETRTO. THE EUROPEAN TYRE AND RIM TECHNICAL ORGANISATION. Standard manual. Belguim, 2001.

MÁRQUEZ, L. Neumáticos agrícolas. Revista Agrotécnica, Torrelodones, p,44-49, 46-52, fev/mar, 2000 .

MÁRQUEZ, L. La elección de los neumáticos. Revista Agrotécnica, Torrelodones, p.41-46, 51-54, fev/mar, 2002.

ORLANDI, A. Il sistema suolo-vehicolo nel motto fuori strada. Bologna: Università degli Studi di Bologna, 1972.

WISMER, R.D.; LUTH, H.J. Prediction of wheel and track performance, 1971. (Technical

Memorandum, 2). documento de difusión restringida 